\title{
Räumliche Darstellung von Klimadaten
}

\section{Einleitung}

Das in den letzten Jahren stetig gewachsene Umweltbewußtsein hat dazu geführt, daß das Klima als Bestandteil unserer natürlichen Umwelt vermehrt in die damit verbundenen Überlegungen und Aktivitäten miteinbezogen wird. Das steigende Interesse, welches von den Umweltwissenschaften an die Klimatologie herangetragen wird, führt zu einer Erweiterung und einer Vertiefung von klimatologischen Forschungsaufgaben.

Im Rahmen des Projektes "Klimaatlas der Schweiz» wird der Versuch unternommen, solchen klimatologischen Fragestellungen nachzugehen und die Forschungsergebnisse in Form eines Kartenwerkes zu veröffentlichen. Es ist vorgesehen, sowohl kleinmaßstäbliche Klimaübersichtskarten für einzelne Klimaelemente im nationalen Raster als auch regionale Klimauntersuchungen für typische Räume unseres Landes in größeren Maßstäben darzustellen.

Das bestehende Stationsnetz der Schweizerischen Meteorologischen Anstalt ergibt ausreichendes Beobachtungsmaterial für die Bearbeitung von allgemeinen Klimaübersichtskarten. Angewandte Klimakarten, z. B. für die Bedürfnisse der Raumplanung, welche auf regionaler Basis zu bearbeiten sind, erfordern jedoch zusätzliche geländeklimatologische Datenerhebungen mit einem erweiterten, engmaschigen Beobachtungsnetz.

Der eine Atlasteil, welcher die Klimaübersichtskarten enthalten wird, umfaßt die Hauptkapitel: Großwetterlagen, Luftdruck, Wind, Strahlung, Temperatur, Feuchtigkeit, Bewölkung, Nebel, Niederschlag, Gewitter, Verdunstung, Phänologie und Klimageschichte. Diese Klimakarten stellen weitgehend analytische Karten dar, welche im allgemeinen die räumliche Analyse eines einzelnen Klimaelementes zum Inhalt haben.

In letzter Zeit hat die Nachfrage nach angewandten Klimakarten für lufthygienische Fragen, nach Klimaeignungskarten für die Landwirtschaft, Siedlung, Tourismus, Industrie und Verkehr sowie nach lokalklimatischen Studien für Planungszwecke stark zugenommen. Hier handelt es sich vorwiegend um synthetische Karten, die praktisch einer Klimaklassifikation für einen bestimmten Anwendungszweck entsprechen, d. h. daß sie für verschiedene Nutzungsarten nach speziellen Kriterien gesondert zu entwer- fen sind. Mit einem Teilprojekt werden solche klimatologische Regionaluntersuchungen durchgeführt und in großmaßstäblichen Klimakarten dargestellt.

Das Atlasprojekt ist als Gemeinschaftswerk konzipiert, welches von verschiedenen Bundes- und Hochschulinstituten getragen wird. Neben der Schweizerischen Meteorologischen Anstalt sind insbesondere das Geographische Institut der Universität Bern und das Bundesamt für Landestopographie, das den Druck und den Verlag übernommen hat, daran beteiligt.

Der vorliegende Bericht gibt einen Überblick über klimatologische Grundlagen, über Messung und Aufbereitung von Klimadaten sowie über grundsätzliche Aspekte im Hinblick auf eine räumliche Darstellung von Klimaelementen. Die angeführten Sachfragen werden anschließend anhand eines Beispiels aus dem Klimaatlas für das Klimaelement Temperatur ausführlich dargelegt.

\section{Klimatologische Grundlagen}

Das Klima ist der langfristige Aspekt des Wetters und wird für einen Ort, eine Landschaft oder für noch größere Räume dargestellt. Die Elemente, die beim Klima betrachtet werden, sind dieselben wie beim Wetter, nämlich Strahlung, Luftdruck, Wind, Lufttemperatur, Luftfeuchtigkeit, Bewölkung und Niederschlag. Die Definition des Klimas unterscheidet sich deutlich von derjenigen des Wettergeschehens, indem sie eine Zusammenfassung der atmosphärischen Vorgänge über einen längeren Beobachtungszeitraum beinhaltet. Die auf statistischen Maßzahlen wie Mittelwert, Streuung, Häufigkeit und Extreme aufbauende Klimadarstellung stützt sich auf meßbare Klimaparameter, die sogenannten Klimaelemente. Da man bei der Beschreibung des Klimas vom kurzfristigen Wettergeschehen abstrahiert, sollte der Zeitraum, der zur Darstellung einer Klimaperiode verwendet wird, hinreichend lang sein, um statistisch gesicherte Angaben zu erhalten. Die Beobachtungsperiode für eine brauchbare Mittelbildung kann für verschiedene Klimaelemente unterschiedlich lang sein. Bei Elementen mit großer Variabilität, wie Temperatur und

Dr. W. Kirchhofer, Schweiz. Meteorologische Anstalt Krähbühlstraße 58, 8044 Zürich 
Niederschlag, sollte sie mindestens 30 Jahre umfassen, wobei man ganz allgemein im Gebirge eine längere Beobachtungsreihe ansetzen sollte als im Flachland.

Bei den Wirkungsfaktoren, die unser Klima prägen, unterscheidet man natürliche und anthropogene Faktoren. $\mathrm{Zu}$ den natürlichen Faktoren gehören die Sonnenstrahlung, die Art der Erdoberfläche (Wasser, Festland mit Bodenart und Bodenbedeckung) sowie die Gestalt der Erdoberfläche (Form, Neigung, Exposition, Höhenlage). Die Kenntnis der anthropogenen Faktoren ist wichtig bei Planungsfragen, da sie zu einer Veränderung des lokalen und regionalen Klimas führen können. Hierzu gehören Änderungen der Bodennutzung, wie Zunahme der Bebauungs- oder Besiedlungsdichte (Stadtklima), neue Industriestandorte (Abwärme, Luftbeimengungen), Schaffung von Verkehrsanlagen (Abwärme, Staub, Abgase), Abholzungen, Aufforstungen, Be- und Entwässerung, Kultivierung (Änderung des Wärmeumsatzes, des Wasserhaushaltes und des Windfeldes).

Eine Klassifikation des Klimas kann aufgrund definierter Grenz- und Andauerwerte verschiedener Klimaelemente vorgenommen werden. Solche Klimaklassifikationen können sich durch klar definierte $\mathrm{K}$ limagrößen an meßbaren Klimaelementen orientieren. In jüngster Zeit sind mit der Entwicklung der synoptischen Meteorologie und der Erforschung der allgemeinen Zirkulation der Atmosphäre genetische Klimaklassifikationen entwickelt worden. Diese modernen Klimaeinteilungen lassen sich jedoch nur schwer quantitativ durch am Ort meßbare Grenz-, Schwellen- oder Andauerwerte klimatischer Elemente fassen.

Im regionalen Bereich ist eine feine, sich an den Gegebenheiten des betreffenden Raumes orientierende Beschreibung notwendig. Ein auf diese Weise definiertes Regionalklima, auch als Landschafts- oder Geländeklima bezeichnet, ist jedoch nur für ein eng begrenztes Gebiet charakteristisch. Es ist einleuchtend, $\mathrm{da} \beta$ die Bestimmung der Eigenschaften und Eigenarten des Regionalklimas ein besonderes Beobachtungsinstrumentarium, insbesondere eine geringe Maschenweite des Beobachtungsnetzes, voraussetzt. Zusätzliche Datenerhebungen bedingen einen großen meßtechnischen Aufwand sowie erhebliche Mehraufwendungen bei der Aufbereitung des Datenmaterials. Auch ein großer Mehraufwand bezüglich Meßvorkehrungen und Datenaufbereitung darf trotzdem nicht darüber hinwegtäuschen, wie großzügig im Grunde genommen unsere klimatologischen Grundlagen bei einer flächendeckenden Darstellung für räumlich ausgedehntere Bereiche sind.

\section{Messung und Aufbereitung von Klimadaten}

Die Grundlage für die Gewinnung unserer Klimadaten bildet das klimatologische Beobachtungsnetz der
Schweiz, bestehend aus rund $120 \mathrm{~K}$ limastationen. Um die Vergleichbarkeit der Beobachtungsdaten zu gewährleisten, sollte eine Beobachtungsstation für einen möglichst großen Umkreis repräsentative Daten liefern. Die Anlage einer Station ist somit wo möglich auf einer freien, ebenen Fläche und abseits lokaler topographischer Besonderheiten zu plazieren. Zudem sind die Datenerhebungen mit Hilfe der meteorologischen Meßinstrumente außerhalb der bodennahen Störungszone durchzuführen. Die Standardausrüstung einer modernen Klimastation erlaubt die laufende Erfassung der Klimaelemente Luftdruck, Wind nach Richtung und Stärke, Strahlung, Lufttemperatur, Extremtemperaturen, Sonnenschein, Luftfeuchtigkeit, Bewölkung, Verdunstung und Niederschlag.

Um die Vergleichbarkeit des klimatologischen Datenmaterials zu gewährleisten, müssen die Umstände, unter denen die Daten an den verschiedenen Beobachtungsstationen gewonnen werden, so weit wie möglich standardisiert werden. Dazu gehört, daß die Meßinstrumente hinsichtlich ihrer Instrumentenkonstanten übereinstimmen. Zudem müssen die Beobachtungen zu bestimmten Klimaterminen ausgeführt werden. Das methodische Prinzip dafür ist, durch Auswahl weniger, aber besonders geeigneter Tagesstunden Werte zu gewinnen, die eine bestmögliche Erfassung der tagesperiodischen Veränderung der Klimaelemente ermöglichen. Die ursprünglich festgelegten drei Beobachtungstermine sind so gewählt, daß mit Hilfe der daraus gewonnenen Temperaturwerte eine größtmögliche Näherung an das 24stündige Tagesmittel erreicht werden kann. Praktische Zwänge haben jedoch zu Kompromissen zwischen den sachlich besten und den für die Beobachter zumutbaren Beobachtungszeiten geführt.

Das gewonnene Beobachtungsmaterial wird in einem ersten Schritt einer Qualitätskontrolle unterzogen; fehlende und falsche Werte müssen ersetzt werden. Aus den gewonnenen Daten lassen sich für Zeitabschnitte wie Tag, Monat oder Jahr durch arithmetische Mittelbildung langjährige klimatologische Mittelwerte sowie weitere statistische Repräsentationsgrößen bestimmen. Für die Vergleichbarkeit des Datenmaterials ist auf die Zahl der Beobachtungsjahre und den Ausschnitt aus der Jahresfolge, auf welche sich die statistischen Werte für die einzelnen Klimaelemente beziehen, zu achten. Im Normalfall sind die Beobachtungsreihen meist ungleich lang und umfassen unterschiedliche Zeitabschnitte. Daraus ergeben sich Probleme der minimalen Periodenlänge sowie der Reduktion der klimatologischen Rechenwerte auf eine einheitliche Bezugsperiode.

Wie lang eine Beobachtungsreihe sein muß, um repräsentative Werte zu liefern, hängt ab von der mehr oder weniger großen Veränderlichkeit des jeweiligen Klimaelementes im Ablauf der Zeit. Wie stark die einzelnen Beobachtungswerte einer Reihe voneinander abweichen, hängt $a b$ von den meteorologischen 
Prozessen, mit denen die Elementenwerte zusammenhängen. Die Variabilität einzelner Klimaelemente ist zudem stark abhängig von der Lage einer Beobachtungsstation. Im allgemeinen zeigt der Niederschlag die größten, die Luftfeuchtigkeit die kleinsten zeitlichen Unregelmäßigkeiten. $\mathrm{Zu}$ den klassischen Darstellungsgrößen einer Beobachtungsreihe gehören neben den Tages-, Monats- und Jahresmittelwerten, den Standardabweichungen und Variabilitäten noch die mittleren und die absoluten Extremwerte für die entsprechenden Zeitabschnitte. Die Differenz zwischen den mittleren Extremwerten wird als mittlere, diejenige zwischen dem tiefsten und dem höchsten Meßwert als absolute Schwankungsbreite einer Meßreihe bezeichnet. Die Größe des Schwankungsbereiches ist abhängig von der Länge einer Zeitreihe.

Bei allen Vorzügen von Mittelwerten darf man nicht übersehen, daß sie eine rechnerisch ermittelte Größe darstellen, die selbst bei Hinzufügen von extremer und mittlerer Schwankungsbreite die realen Gegebenheiten nur unvollkommen wiederzugeben vermag. Ein Monat, der beispielsweise in der ersten Hälfte gleichmäßig mild, in der zweiten Hälfte jedoch anhaltend kalt ist, hat dieselben mittleren und extremen Werte wie einer mit permanentem, tagesperiodischem Wechsel zwischen milden Mittags- und kalten Nachttemperaturen, obwohl dies für klimatologische Folgeerscheinungen unterschiedliche Konsequenzen hat.

\section{Darstellung von Klimakarten}

In einer Klimakarte werden klimatologische Zusammenhänge räumlich dargestellt. Von den einzelnen Stationen ausgehend, werden die Klimadaten entsprechend ihrer regionalen Höhenabhängigkeit auf den gesamten Raum übertragen, und zwar mit stetigen, fließenden Übergängen. Es handelt sich also um die kartographische Darstellung von Klimaelementen in Form von thematischen Karten, wobei auch die topographischen Gegebenheiten, welche das Verhalten der einzelnen Klimaelemente wesentlich beeinflussen, in der Kartendarstellung zu berücksichtigen sind. Die topographische Karte bildet somit die räumliche Voraussetzung und das Basisgefüge für jeden klimatologischen Kartenentwurf.

Bevor man mit dem eigentlichen Kartenentwurf beginnt, sollten Vorabklärungen über Dinge wie Gebietsabgrenzung, Inhalt, Darstellungsart, Format und Maßstab angestellt werden. Die Abklärungen solcher Vorfragen sind wesentlich für das Gelingen einer Klimakarte und können jeweils nur durch gegenseitiges Abwägen getroffen werden. Vor Beginn einer Kartenzeichnung schafft man sich Klarheit über die graphische Gliederung des Inhalts, welche ihren Niederschlag in der Kartenlegende findet. In der Formulierung und Gliederung der Kartenlegende steckt ein wesentlicher Teil der konzeptionellen
Vorarbeit. Legendenaufstellung sowie die erforderlichen Zusammenfassungen sind unter Mitberücksichtigung des Kartenmaßstabes vorzunehmen. Der Maßstab ist ein wesentliches Charaktermerkmal jeder Karte, wobei für Arbeitskarten und Originalentwürfe in der Regel größere Maßstäbe zu verwenden sind, als für die Herausgabe geplant ist. Der Maßstab ist abhängig vom darzustellenden Gebiet, vom Thema und dessen Gliederung, vom Forschungsstand und vor allem vom Verwendungszweck der Klimakarte. Nicht selten findet man Klimakarten mit unnötig großen Maßstäben. Solche Karten täuschen im allgemeinen eine Genauigkeit vor, die dem Wissensstand über die klimatischen Gegebenheiten nicht entspricht. $\mathrm{Zu}$ große Maßstäbe verlocken zu graphischer Differenzierung von Dingen, die man zu wenig kennt. Einfach gegliederte und nicht genau erfaßbare geophysikalische Erscheinungen bedürfen in Klimaübersichtskarten keiner allzugroßen Maßstäbe. Für Karten der mittleren Lufttemperatur oder der mittleren Jahresniederschläge z. B. genügen in der Regel Maßstäbe 1:1 Mio. oder kleiner.

Jeder klimatologische Inhalt ist eingebettet in einem topographischen Kartengefüge. Der Autor einer Klimakarte sollte sich daher schon bei Beginn seiner zeichnerischen Originalerstellung über die Wahl der topographischen Grundkarte im klaren sein. Diese Basiskarte hat das ihr eingefügte Thema möglichst zu stützen und sinnvoll zu ergänzen, doch soll sie es anderseits möglichst wenig belasten. Für kleinmaßstäbliche Klimakarten genügen in der topographischen Basiskarte die Elemente Gewässernetz und Stationsbezeichnungen, verbunden mit einem einfachen Relief-Schattenbild. Ein solches Relief erhöht die Übersichtlichkeit durch einfache, räumliche Gliederung und dient sowohl der Lagezuordnung als auch dem besseren klimatologischen Verständnis. Die Elemente der Basiskarte haben sich dem klimatologischen Karteninhalt graphisch zu unterordnen. Meist empfiehlt es sich, die Basiselemente grau zu drucken. Bunte Farben, starke Töne und kräftige Signaturen bleiben dem Thema vorbehalten.

Die räumliche Verteilung der Klimaelemente wird vorwiegend in Form von Isolinienkarten dargestellt. Eine Isolinie verbindet benachbarte Punkte gleicher Werte oder gleicher Intensität des dargestellten Klimaparameters. Sind sie in eine Geländedarstellung eingefügt, so gibt eine solche Karte Auskunft über die geometrische Lage, verbunden mit dem thematischen Wert, z. B. dem lokalen Temperaturmittel. Die Isolinien ergänzen somit gleichsam das Bild des topographischen durch das Bild des thematischen Reliefs. Bei den Klimaelementen handelt es sich, wie bei den meisten naturgegebenen Kontinua, um schwankende, sich rasch oder langsam ändernde Erscheinungen. Die kartierten Ergebnisse sind in der Regel Mittelwerte aus kürzeren oder längeren Beobachtungsreihen. Ihre Zuverlässigkeit hängt ab von der Dichte der Meßstationen, der Qualität und Anzahl der Messungen und 
vor allem auch von der Zeitdauer einer Meßperiode. Die anzustrebende Aufnahmegenauigkeit einer Isolinienkarte ist abhängig von der Art des Kontinuums und von den Ansprüchen, die man an eine Karte stellt. Die Genauigkeit gewisser Isolinien kann gesteigert werden durch systematische Auswertung lokaler Relationen zwischen thematischem und topographischem Relief. Eine gute Kenntnis dieser Relationen erleichtert ein Urteil über Qualität und Genauigkeit einer Isolinienkarte. Bei geophysikalischen Erscheinungen überschreitet die Lageunsicherheit der Linien in Karten großer Maßstäbe oft ein Mehrfaches der horizontalen Linienabstände. Solche Isolinien sind daher meist nur in Karten kleiner Maßstäbe sachlich vertretbar. In Karten mit mittleren und kleineren Maßstäben drängen sich generalisierende Linienvereinfachungen auf. Ausmaß und Art jeder generalisierenden Vereinfachung hängen vor allem ab vom Formencharakter, von der Aufnahmegenauigkeit, vom Kartenmaßstab und von der Größe der thematischen Wertstufen. Bei klimatologischen Erscheinungen wäre es nicht sinnvoll, die Isolinien sehr fein gliedern zu wollen, denn es handelt sich hierbei nicht um feste, exakt erfaßbare Dinge, sondern um Mittelwerte aus Beobachtungsreihen veränderlicher $\mathrm{Zu}$ stände.

Bei der Herstellung von Originalkarten sind Anzahl und Wahl der zu verwendenden Flächenfarbtöne eingeschränkt durch die Anforderung guter Unterscheidbarkeit. Die Zuweisung von Farbtönen erfolgt vorwiegend nach inhaltlichen Gesichtspunkten, nach Art und Bedeutung der graphischen Gestaltung. Im allgemeinen soll danach getrachtet werden, mit einer möglichst kleinen Anzahl von Druckfarben ein möglichst ausdruckstarkes, farblich gut differenziertes Bild zu gestalten. In Klimakarten ist es selten möglich, farbige Naturähnlichkeit zu erreichen. In manchen Fällen wählt man gebräuchliche oder typisch erscheinende Farben. In Temperaturkarten kann z. B. RotOrange-Gelb für warm, Blau-Grün für kalt angewendet werden. Wichtiges oder Außergewöhnliches soll hervortreten, Allgemeines und weniger Wichtiges nur mitklingen. Eine Karte kann als gut gewertet werden, wenn darin der klimatologische Inhalt in einfacher, deutlicher Form zum Ausdruck gebracht wird.

Im Gegensatz zu den synoptischen Wetterkarten ist es für die Bearbeitung von klimatologischen Karten sehr schwierig, grenzübergreifende, einheitliche Datensätze von klimatologischen Meßreihen zu erhalten. Eine solch grenzübergreifende Datenaufbereitung wäre nur mit sehr großen Mehraufwendungen zu bewerkstelligen, so daß man im allgemeinen die klimatologische Bearbeitung nicht über die politischen Grenzen hinweg ausdehnt. Auch die Karten unseres Klimaatlasses machen diesbezüglich keine Ausnahme, sie werden aus diesen Gründen thematisch als Inselkarten dargestellt. Die topographische Grundlagekarte, in welche das klimatologische Thema eingebettet ist, wird zur besseren Übersicht über die
Landesgrenze hinaus erweitert. Die vorliegenden Klimaübersichtskarten werden also gesamthaft als Rahmenkarten dargestellt.

\section{Räumliche Darstellung der Temperatur}

\subsection{Temperaturmessung}

Unter den Klimaelementen stellt die Lufttemperatur, welche primär über den Strahlungshaushalt zustande kommt, ein wichtiges Basiselement dar. Eine mitgestaltende Rolle spielen der horizontale und vertikale Luftmassenaustausch, das Relief nach Höhe und Exposition sowie die natürliche und vom Menschen geschaffene Bodenbedeckung als geographische Einflußfaktoren.

Für klimatologische Zwecke wird die Lufttemperatur im allgemeinen in einer genormten, gut belüfteten Wetterhütte, zwei Meter über dem Erdboden, in Grad Celsius gemessen. Auf diese Weise können die Thermometer gegen direkte und indirekte Strahlung sowie gegen den Einfluß des Untergrundes geschützt werden. Mit dieser Meßanordnung erhalten wir repräsentative Meßwerte, welche bei normalen Verhältnissen für einen größeren räumlichen Bereich Gültigkeit haben. Die Klimastationen messen die Morgen-, Mittag- und Abendtemperaturen mit einem Stationsthermometer. Die Extremtemperaturen werden mit speziellen Minimum- und Maximumthermometern ermittelt. Um wirkliche Vergleichbarkeit zu erreichen, erfolgen die Ablesung und weitere rechnerische Aufbereitung der Klimadaten nach möglichst einheitlichen Normen. Das Tagesmittel der Lufttemperatur kann aus den drei Terminmessungen nach der Formel $(07.30+13.30+2 \times 21.30) / 4$ berechnet werden. Aus den einzelnen Tagesmittelwerten lassen sich die Monats- und Jahresmittelwerte, bei Zusammenfassung mehrerer Jahre die langjährigen klimatologischen Mittelwerte bestimmen.

\subsection{Bearbeitung langjähriger Temperaturreihen}

Die Darstellung der räumlichen Verteilung eines Klimaelementes verlangt abgesichertes; statistisches Datenmaterial. Wie erwähnt, muß die Meßreihe eine um so längere Periode aufweisen, je größer die Schwankungsbreite des zu betrachtenden Klimaelementes ist. Für die Bearbeitung der Temperaturkarten werden die langjährigen Meßreihen des klimatologischen Beobachtungsnetzes für den Zeitraum 1931-1970 herangezogen. In einem solch langen Zeitraum beobachten viele Klimastationen nicht durchgehend. Durch Ausscheiden der Beobachter oder aus anderen Gründen mußten Stationen verlegt oder gar aufgelöst werden. Da aber nur homogenes Datenmaterial vergleichbar ist, sind die inhomogenen und kürzeren Meßreihen auf die Basisperiode 1931-1970 umgerechnet worden. 
Als Vergleichsstationen, wie sie für die Homogenisierung der Datensätze benötigt werden, dienen Klimastationen, welche über den gesamten Zeitraum von 1931-1970 möglichst homogene Temperaturreihen aufweisen. Als Reduktionsverfahren ist im vorliegenden Fall die Differenzenmethode angewendet worden. Mit Hilfe der Temperaturdifferenzen bezüglich zweier oder mehrerer Klimastationen desselben Klimagebietes können inhomogene Meßreihen ausgeglichen bzw. auf eine einheitliche Periodenlänge umgerechnet werden. Derartige Verfahren können - abgesehen vom Problem der Meßgenauigkeit - weitere Fehlermöglichkeiten bedingen, da die Vergleichsstationen, infolge der Dichte des Stationsnetzes, vielfach mehr oder weniger weit entfernt liegen. Hinzu kommt das abweichende Verhalten im Gebirgsland, wo z. B. Tal-, Hang- und Gipfellagen einen unterschiedlichen Tagesund Jahresgang der Lufttemperatur aufweisen. Aus diesen Gründen muß man den Mittelwerten eine gewisse Streubreite zuerkennen und dies bei der Festlegung der Isothermen entsprechend berücksichtigen.

\subsection{Regionale Temperaturdifferenzierung}

Eine wichtige Aufgabe der Klimatologie ist die Übertragung der örtlich gemessenen und statistisch verarbeiteten klimatologischen Daten auf den Raum. Dieses Teilgebiet der regionalen Klimatologie beschäftigt sich vorwiegend mit den Mittel- und Extremwerten sowie der Streuung und Häufigkeitsverteilung einzelner Klimaelemente.

Die räumliche Darstellung der Wärmeverhältnisse in einem bestimmten Gebiet geschieht durch Isothermen. Die Zuverlässigkeit einer Isothermenkarte ist sehr unterschiedlich, weil die Dichte des Stationsnetzes wechselt und außerdem die Zuverlässigkeit der einzelnen Werte variiert. Eine Isothermenkarte wird der Übersicht und der Vergleichbarkeit halber über etwelche Lücken und Inhomogenitäten hinwegtäuschen.

Man kann die Isothermen nach wirklichen, unreduzierten Werten konstruieren, die als Mittelwertskarten ohne weitere Veränderung aus den registrierten Meßdaten gewonnen werden und somit das tatsächlich anzutreffende Wärmeverteilungsbild wiedergeben. Die vertikalen Temperaturunterschiede sind im Verhältnis zu den horizontalen Differenzen in gleicher Meereshöhe so bedeutend, daß diese unreduzierten Isothermen fast identisch sind mit den Höhenkurven. Es besteht auch die Möglichkeit, die Temperaturwerte auf ein Einheitsniveau, z.B. auf Meeresniveau, zu reduzieren. Die Reduktion auf ein Bezugsniveau, mit den für das betreffende Gebiet und die entsprechende Jahreszeit gültigen vertikalen Temperaturgradienten, ergibt ein Bild über die Temperaturverteilung in dieser Bezugshöhe: Die vorliegenden Temperaturkarten der Monats- und Jahresmittel basieren auf den unreduzierten Originalbeobachtungen. Diese Originalwerte wurden in eine Kartenunterlage mit Höhenlinien eingetragen und dann der Verlauf der Isothermen festgelegt. Diese Methode gewährleistet ein nach einheitlichen Gesichtspunkten durchgearbeitetes Kartenbild.

Bei der Festlegung der Wertstufen für die Isothermen sind im wesentlichen die folgenden drei Gesichtspunkte beachtet worden:

- Vergleichbarkeit sämtlicher Monats- und Jahrestemperaturkarten;

- Gute räumliche Temperaturabstufung sowohl im Flachland als auch im Gebirge;

- Sinnvolle Genauigkeit bezüglich Stationswert, Stationsdichte, Lagefehler und horizontalen Linienabstands der Isothermen.

Tabelle 1 Mittelwerte ( $\overline{\mathrm{x}})$ und Standardabweichungen (s) der Temperatur $\left({ }^{\circ} \mathrm{C}\right)$ für die Stationen Säntis (2500 m), Zürich (556 m), Davos (1580 m) und Lugano (275 m); Zeitraum 1931-1970.

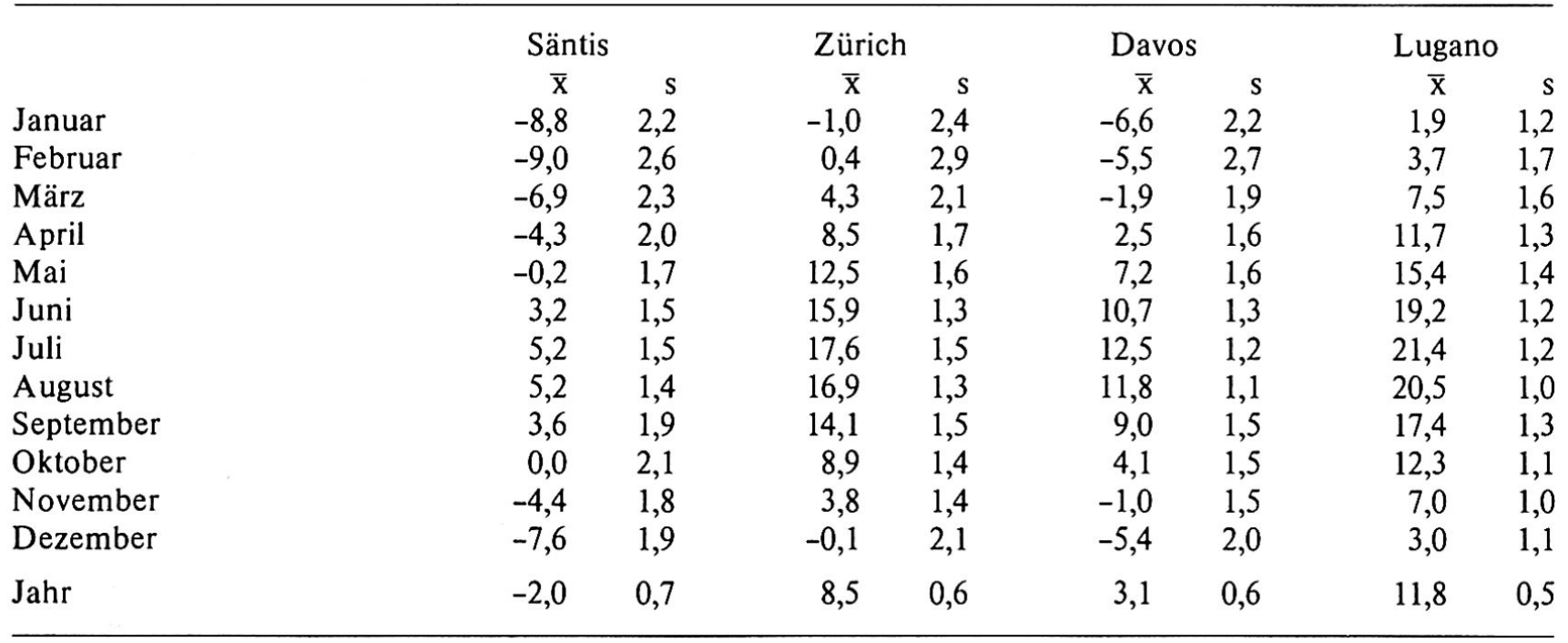




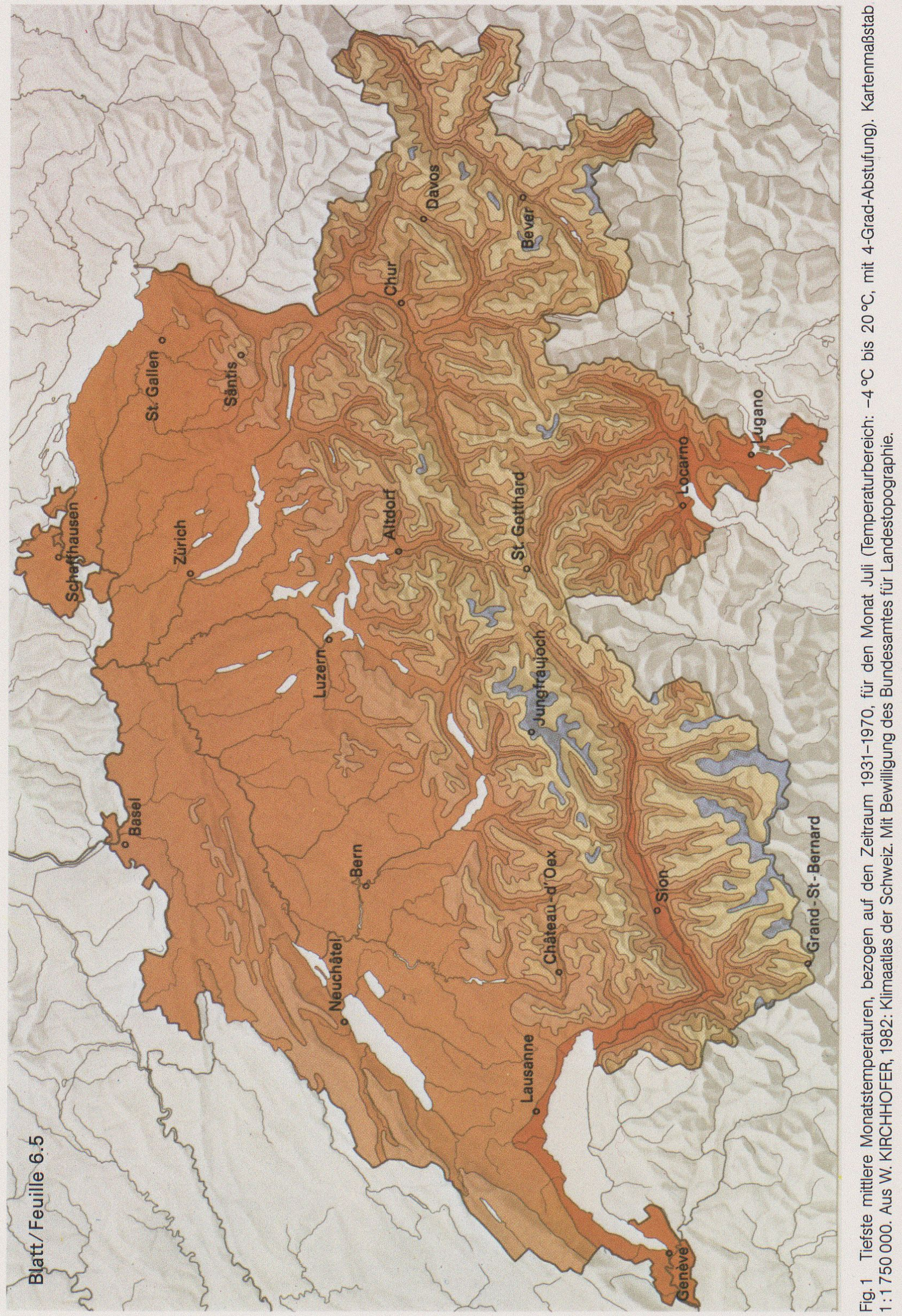



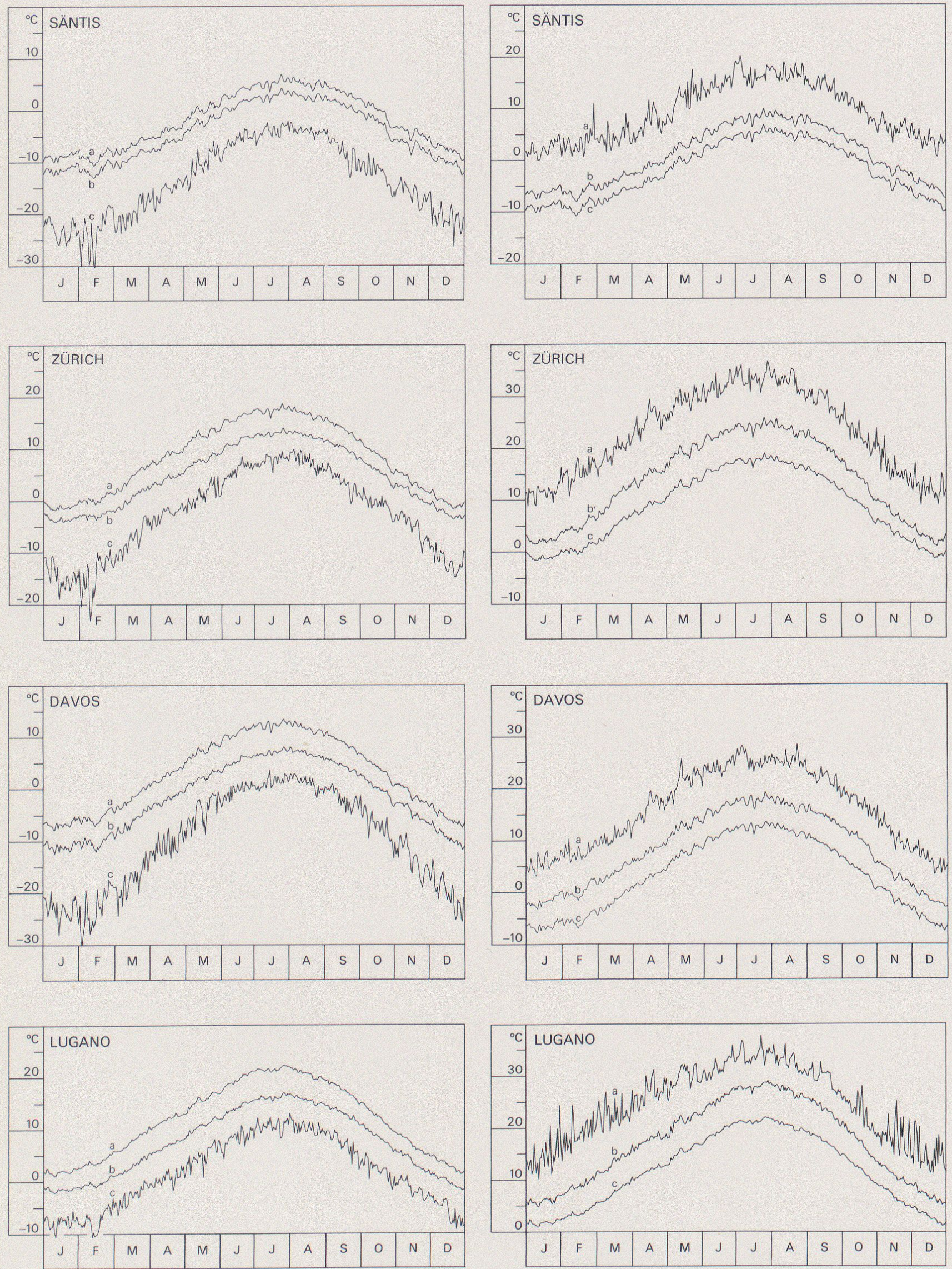

Fig. 2 Temperaturreihen 1931-1970, Jahresverlauf a: Mittlere Tagestemperaturen

Fig. 3 Temperaturreihen 1931-1970, Jahresverlauf a: Temperatur-Maxima (Einzelwerte)

b: Mittlere Temperatur-Minima

b: Mittlere Temperatur-Maxima

c: Temperatur-Minima (Einzelwerte)

c: Mittlere Tagestemperaturen 
Ein einheitliches Verfahren zur Festlegung von Wertstufen für Isolinien läßt sich schwer entwickeln. Für die vorliegenden Temperaturkarten ist eine statistische Maßzahl, nämlich die Standardabweichung der Monats- bzw. der Jahresmittelwerte, beigezogen worden. In Tabelle 1 sind für die Klimastationen Säntis, Zürich, Davos und Lugano die Monatsund Jahresmittelwerte $(\overline{\mathrm{x}})$ sowie die entsprechenden Standardabweichungen (s) der Mittelwertreihen für den Zeitraum 1931-1970 zum Vergleich aufgeführt.

Unter der Annahme, daß die Abweichungen der Mittelwerte der einzelnen Jahre um die langjährigen Mittelwerte $(\bar{x})$ eine Normalverteilung aufweisen, liegen etwa $95 \%$ aller dieser Mittelwerte für die einzelnen Stationen zwischen $\bar{x}-2 s$ und $\bar{x}+2 s$. Wie das Zahlenbeispiel in Tabelle 1 zeigt, sind die Standardabweichungen (s) auch für ganz unterschiedliche Gebiete im Mittel kleiner als $2^{\circ} \mathrm{C}$.

Mit der Forderung, daß die um eine Isotherme streuenden Mittelwerte mit einer Wahrscheinlichkeit von ca. $95 \%$ die Nachbarisothermen nicht überschreiten dürfen, ist für die Temperaturabstufung ein einheitlicher Wert von $4^{\circ} \mathrm{C}$, nämlich der 2 fache mittlere Maximalwert der Standardabweichung, festgelegt worden. Mit dieser Wertabstufung der Isothermen ist es möglich, die obengenannten Gesichtspunkte ausgewogen auf die Temperaturkarten zu übertragen. Zur besseren räumlichen Differenzierung bzw. Auflösung wird in gewissen Fällen von der Möglichkeit Gebrauch gemacht, innerhalb der Farbstufen eine $\mathrm{Z}$ wischenisotherme zu legen.

In Figur 1 ist die Julikarte der tiefsten, mittleren Monatstemperaturen aus dem «Klimaatlas der Schweiz» dargestellt. Bei diesen Temperaturkarten werden stationsweise die tiefsten Monatsmittelwerte bestimmt und zu Monatskarten zusammengefaßt.

Wie bereits erwähnt, unterliegt die räumliche Verteilung der Klimaelemente verschiedenen Einflüssen, wobei in unserem Raum die Höhe und Geländeform eine wesentliche Rolle spielen. Beim Entwerfen von Klimakarten ist somit eine gründliche Analyse der Meßwerte sowie die Kenntnis der Geländeverhältnisse erforderlich. Ein gewisser Anteil Subjektivitàt in der Linienführung ist so oder so in jeder Karte enthalten. Aus diesem Grunde sollte in einer Klimakarte die Lage der Isolinien nur als Orientierungshilfe verstanden werden. Sie grenzen Gebiete gegeneinander $a b$, in denen das jeweilige Klimaelement mehr oder weniger um einen mittleren Wert streut. Trotz aller in den Karten erkennbaren Strukturen in der Linienführung handelt es sich um generalisierte Isolinien, die nicht auf einen anderen Maßstab vergrößert werden dürfen. Die Isolinien sind als fließender Übergang mit einer gewissen Bandbreite zu werten. Sie dienen der räumlichen Unterscheidung und sind keine fest fixierten Grenzen. Punktinformationen sollten im allgemeinen nicht aus Karten, sondern aus Klimatabellen entnommen werden.

\subsection{Vertikale Temperaturverteilung}

Im Mittel besteht ein Wärmegefälle von den bodennahen zu den höheren Luftschichten. Die Lufttemperatur nimmt also in der Regel mit zunehmender Höhe ab. Das Maß für die vertikale Temperaturabnahme ist der vertikale Temperaturgradient, ausgedrückt in Grad Celsius pro $100 \mathrm{~m}$. Der vertikale Temperaturgradient kann entweder durch Vergleich benachbarter Stationen mit großem Höhenunterschied (Berg- und Talstationen) oder durch direkte aerologische Messungen ermittelt werden. Die Messungen der Lufttemperatur an verschieden hoch gelegenen Klimastationen zeigen im Durchschnitt eine ähnliche Temperaturabnahme mit der Höhe wie diejenigen in der freien Atmosphäre. Allerdings werden die Werte durch verschiedene Faktoren wie Hanglage. Muldenlage, Lee- oder Luvseite zur Hauptwindrichtung modifiziert.

Die Größe der vertikalen Temperaturabnahme unterliegt ähnlichen Schwankungen wie die räumliche Verteilung der Temperatur in der horizontalen Ausdehnung und weist sowohl regionale als auch jahreszeitliche Unterschiede auf. Im Winter liegen die Gradientwerte etwas tiefer als im Sommer, dies als Folge der häufigen Inversionsbildung während der kalten Jahreszeit. Die in der Schweiz auftretenden Gradientwerte zeigen Werte um $0,5^{\circ} \mathrm{C} / 100 \mathrm{~m}$. Im Winter liegen sie, abgesehen von der Alpensüdseite, um ca. $0,1^{\circ} \mathrm{C}$ (im Jura bis $0,2^{\circ} \mathrm{C}$ ) tiefer. Im Frühling und Sommer nehmen sie in allen Landesteilen Werte um $0,6^{\circ} \mathrm{C} / 100 \mathrm{~m}$ an. Im Herbst gehen die Werte wieder zurück auf ca. $0,5^{\circ} \mathrm{C} / 100 \mathrm{~m}$, wobei der Jura mit ca. $0,3^{\circ} \mathrm{C} / 100 \mathrm{~m}$ die Ausnahme bildet. Wie bereits erwähnt, basieren die vorliegenden Temperaturkarten auf nicht reduzierten Originalwerten. Demzufolge sind die regional unterschiedlichen Gradientwerte auch in den vorliegenden Temperaturkarten enthalten. Für die Festlegung der Lage der einzelnen Isothermen ist jedoch, unter Berücksichtigung der Originalwerte, ein einheitlicher $\mathrm{Gradientwert}$ von $0,5^{\circ} \mathrm{C} / 100 \mathrm{~m}$ angewendet worden.

\subsection{Langjähriger Temperaturverlauf}

Die Karten der mittleren Monatstemperaturen geben einen Überblick über den mittleren Jahresverlauf der Lufttemperatur. Anhand der tiefsten bzw. höchsten Monatstemperaturkarten werden die mittleren Schwankungsbreiten für die einzelnen Jahreszeiten kartographisch festgehalten. Bei diesen Klimakarten handelt es sich um die räumliche Darstellung von rechnerisch ermittelten Temperaturgrößen, welche die realen Gegebenheiten an einer Einzelstation nur unvollkommen wiederzugeben vermögen.

In den Figuren 2, 3 und 4 sind für die Stationen Säntis, Zürich, Davos und Lugano verschiedene Temperaturwerte im langjährigen Verlauf aus dem «Klimaatlas der Schweiz» dargestellt. Die mittleren Monats- und Jahrestemperaturen, bezogen auf den Zeitraum 1931-1970, basieren auf den Tagesmittelwerten der 

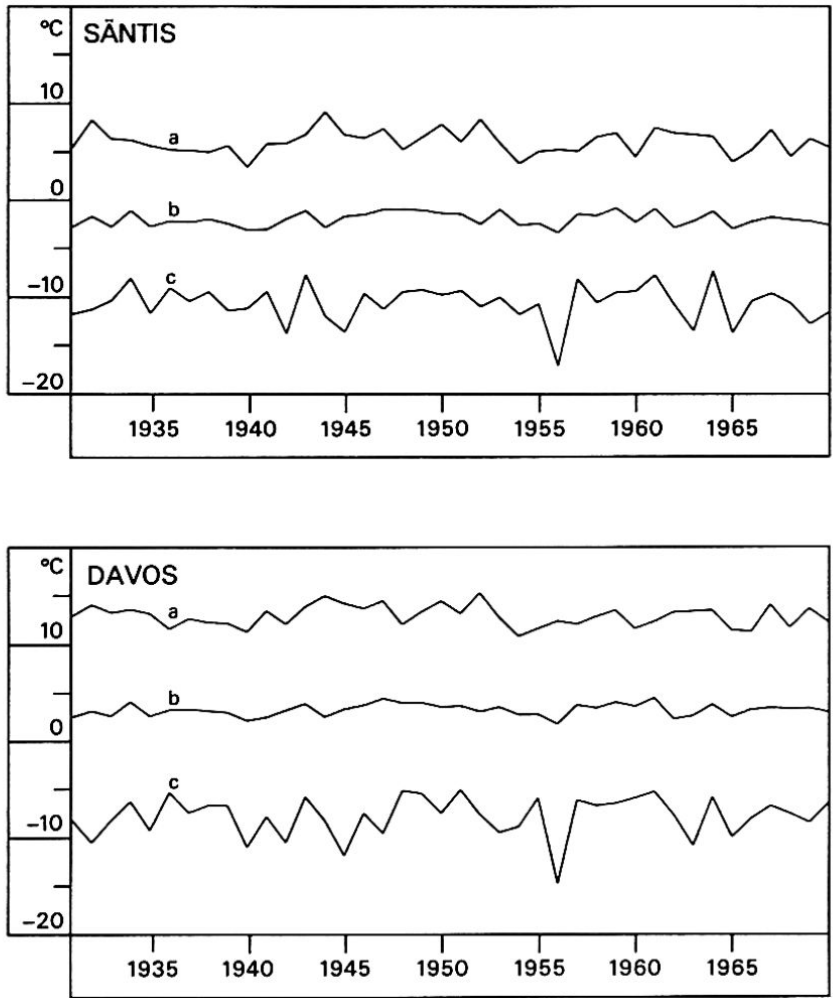

Fig. 4 Temperaturreihen 1931-1970

a: Höchste mittlere Monatstemperaturen

b: Mittlere Jahrestemperaturen

c: Tiefste mittlere Monatstemperaturen

Lufttemperatur, die aus den Terminbeobachtungen nach der Formel $(07.30+13.30+2 \times 21.30) / 4$ berechnet werden. Bei den mittleren Minima- und Maximatemperaturen handelt es sich um gewöhnliche arithmetische Mittelwerte, wie wir sie aus den täglichen Ablesungen der Extremthermometer erhalten.

Mit diesen Beispielen wird aufgezeigt, daß eine Klimakarte, ergänzt mit klimatologischen Punktinformationen, wesentlich an Aussagekraft gewinnt. Auf diese Weise kann sowohl das räumliche wie das zeitliche Verhalten einzelner Klimaelemente übersichtlich dargestellt werden.

\section{Zusammenfassung}

Die Schweizerische Meteorologische Anstalt wird im Rahmen des Werkes «Klima der Schweiz» ein Kartenwerk in Form eines Kimaatlasses herausgeben. Es ist vorgesehen, sowohl kleinmaßstäbliche Klima-
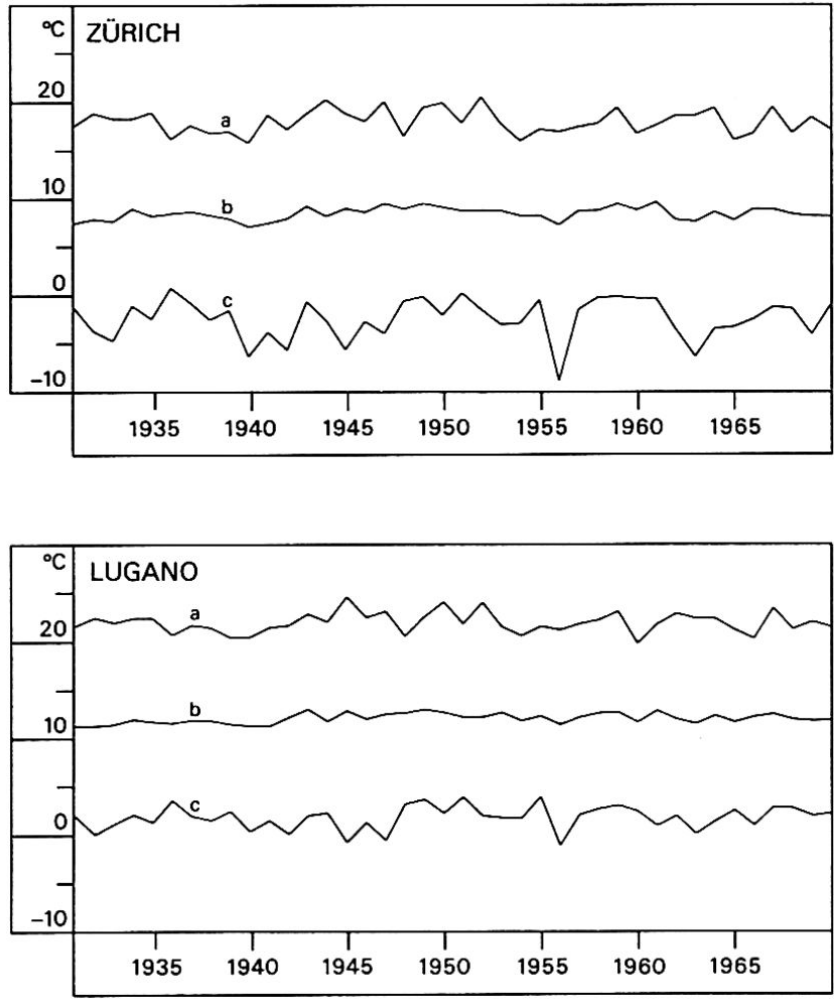

übersichtskarten im nationalen Raster als auch regionale Klimauntersuchungen für typische Räume unseres Landes in größeren Maßstäben darzustellen.

Im vorliegenden Bericht wird das Projekt «Klimaatlas der Schweiz» vorgestellt. Dann folgt ein Überblick über grundsätzliche Fragen, wie sie bei der Bearbeitung langjähriger Meßreihen im Hinblick auf eine räumliche Darstellung auftreten. Die angeführten Sachfragen betreffend Messung, Aufbereitung und Darstellung von Klimadaten werden hernach am Beispiel der Lufttemperatur ausführlich dargelegt.

\section{Literatur}

IMHOF E. (1972): Thematische Kartographie, Berlin.

KIRCHHOFER W. (1982): Klimaatlas der Schweiz; erste Lieferung. Veröffentlichung der Schweizerischen Meteorologischen Anstalt, 40 S. Verlag: Bundesamt für Landestopographie, Wabern. 